\title{
Remarks on time-dependent [current]-density functional theory for open quantum systems
}

\section{Citation}

Yuen-Zhou, Joel, and Alán Aspuru-Guzik. 2013. Remarks on Time-Dependent [current]Density Functional Theory for Open Quantum Systems. Physical Chemistry Chemical Physics 15 (30):12626-12636.

\section{Published Version}

doi:10.1039/c3cp51127h

\section{Permanent link}

http://nrs.harvard.edu/urn-3:HUL.InstRepos:12561404

\section{Terms of Use}

This article was downloaded from Harvard University's DASH repository, and is made available under the terms and conditions applicable to Other Posted Material, as set forth at http:// nrs.harvard.edu/urn-3:HUL.InstRepos:dash.current.terms-of-use\#LAA

\section{Share Your Story}

The Harvard community has made this article openly available.

Please share how this access benefits you. Submit a story.

\section{Accessibility}


Cite this: Phys. Chem. Chem. Phys., 2013, 15, 12626

Received 18th March 2013, Accepted 6th June 2013

DOI: $10.1039 / \mathrm{c} 3 \mathrm{cp} 51127 \mathrm{~h}$

www.rsc.org/pccp

\title{
Remarks on time-dependent [current]-density functional theory for open quantum systems
}

\author{
Joel Yuen-Zhou*ab and Alán Aspuru-Guzik*ab
}

\begin{abstract}
Time-dependent [current]-density functional theory for open quantum systems (OQS) has emerged as a formalism that can incorporate dissipative effects in the dynamics of many-body quantum systems. Here, we review and clarify some formal aspects of these theories that have been recently questioned in the literature. In particular, we provide theoretical support for the following conclusions: (1) contrary to what we and others had stated before, within the master equation framework, there is in fact a one-to-one mapping between vector potentials and current densities for fixed initial state, particleparticle interaction, and memory kernel; (2) regardless of the first conclusion, all of our recently suggested Kohn-Sham (KS) schemes to reproduce the current and particle densities of the original OQS, and in particular, the use of a KS closed driven system, remains formally valid; (3) the Lindblad master equation maintains the positivity of the density matrix regardless of the time-dependence of the Hamiltonian or the dissipation operators; (4) within the stochastic Schrödinger equation picture, a one-to-one mapping from stochastic vector potential to stochastic current density for individual trajectories has not been proven so far, except in the case where the vector potential is the same for every member of the ensemble, in which case, it reduces to the Lindblad master equation picture; (5) master equations may violate certain desired properties of the density matrix, such as positivity, but they remain as one of the most useful constructs to study OQS when the environment is not easily incorporated explicitly in the calculation. The conclusions support our previous work as formally rigorous, offer new insights into it, and provide a common ground to discuss related theories.
\end{abstract}

Time-dependent density functional theory (TDDFT) is a rigorous reformulation of time-dependent quantum mechanics whose central object of study is the particle density rather than the many-body wavefunction. ${ }^{1-4}$ Two important theorems lie at the core of its theoretical foundations. The first one, by Runge and Gross (RG), ${ }^{5}$ establishes a one-to-one mapping from scalar potentials to particle densities. The second one is the $V$-representability theorem due to Van Leeuwen, ${ }^{6}$ which guarantees that the particle density of an interacting system can be reproduced by a non-interacting surrogate system, called Kohn-Sham (KS) system ${ }^{7}$ due to historical reasons in analogy to (time-independent) density functional theory. These two results justify the common practice of TDDFT: first, to use a KS system to compute the particle density evolution and, second, to express the desired observable of the original system (e.g., the dipole moment) as a functional of the particle density.

\footnotetext{
${ }^{a}$ Research Laboratory of Electronics, Massachusetts Institute of Technology, 77 Massachusetts Avenue, 02139, Cambridge, MA, USA. E-mail: joelyuen@mit.edu

${ }^{b}$ Department of Chemistry and Chemical Biology, Harvard University, 12 Oxford

Street, 02138, Cambridge, MA, USA. E-mail: aspuru@chemistry.harvard.edu
}

There is also an analogous theory due to Vignale, ${ }^{8}$ named timedependent current-density functional theory (TDCDFT), which establishes a one-to-one correspondence between vector potentials and current densities, and guarantees the existence of a noninteracting KS system which, evolving under a fictitious KS vector potential, reproduces the current density of the original system. Since the particle density is related to the current density via the continuity equation, the original particle density is also reproduced in such KS system.

The performance of TDDFT depends on the energy functionals one employs and the ability to express the desirable observables in terms of the particle density. However, its role as a practical method to obtain dynamical properties of manybody systems is so far unrivaled by other $a b$ initio methods due to the accuracy it provides with the currently available functionals versus the computational cost it entails. General prescriptions to compute excited state energies and their oscillator strengths via TDDFT have been reported, ${ }^{9-15}$ and have been successfully used to study a wide variety of phenomena, such as chemical ${ }^{16-21}$ and nonlinear optical ${ }^{22-24}$ properties in nanomaterials, resonant energy transfer, ${ }^{25,26}$ and many-body 
effects in solid state systems, ${ }^{27-29}$ and lifetimes of atomic resonance states, ${ }^{30,31}$ among many others. Whereas most of the studies so far have relied on the local density approximation, ${ }^{1,32-34}$ a big effort has also been taken on the direction of capturing memory effects. ${ }^{33-38}$ Furthermore, there has been considerable interest in the extension of TDDFT to study situations where there is an interplay between electronic and nuclear degrees of freedom and both are explicitly included in the simulation. ${ }^{39-45}$ An alternative approach to this problem relies on Open Quantum Systems (OQS) theory, where the electronic degrees of freedom are evolved as a quantum master equation (ME), with the bath of phonons affecting the electrons via fluctuations and dissipation. ${ }^{4-55}$ We hereby restrict the definition of OQS to the domain of systems that exchange energy-but not particles, with an environment. Scenarios where there is an actual exchange of particles between the system and the environment are beyond the scope of this article, but we refer the reader to previous investigations along these lines of thought. ${ }^{56-59}$

The description of OQS is essential in a wide variety of fields, ranging from quantum optics to chemical dynamics in condensed media. ${ }^{60-63}$ In our work on OQS-TDDFT, we have established precise conditions for $\vec{V}$ and $\vec{A}$ representability for the evolution of general ME. ${ }^{51,53,93}$ An interesting outcome of our investigation is that particle and current densities of OQS can be reproduced in closed driven systems (CDS) evolving unitarily. Recently, in an unrefereed posting in the arXiV e-Print server $^{64}$ and its sequel, ${ }^{65}$ D'Agosta and Di Ventra (DADV) have casted doubts on several of our results, potentially generating awareness but also confusion among the practitioners of the field, since it is not clear whether they or we are correct. We regard this occasion as a good opportunity to present what we believe to be an objective account of the subject. The goal of this article is to clarify our work ${ }^{51,53,93}$ in comparison with theirs ${ }^{48,49,66}$ in the broad context of OQS in TDDFT. The paper is structured as follows: in Section 1, we establish the notation which will be used throughout the paper, and in Section 2, we address a series of formal issues of TDDFT for OQS which have been a potential source of confusion in the literature. This work paves the theoretical foundations for further development of practical methods to simulate dynamics of many-body OQS.

\section{Notation}

Consider the general evolution of the density matrix in the form of a ME:

$$
\frac{\mathrm{d} \rho(t)}{\mathrm{d} t}=-i\left[\hat{H}_{\mathrm{S}}(t), \rho(t)\right]+\int_{0}^{t} \mathrm{~d} t^{\prime} \mathcal{K}\left(t, t^{\prime}\right) \rho\left(t^{\prime}\right)+\mathcal{T}(t) .
$$

Here $\rho(t)$ is the density matrix describing the quantum state of the system of interest, $\mathcal{K}\left(t, t^{\prime}\right)$ is a memory kernel which describes how the state at times $0 \leq t^{\prime} \leq t$ affect the dynamics at time $t$. If $\mathcal{K}\left(t, t^{\prime}\right) \propto \delta\left(t-t^{\prime}\right)$, the ME is Markovian; otherwise, it is non-Markovian. $\mathcal{T}(t)$ is an inhomogeneous term that encodes initial system-bath correlations. The discussion of this article does not pertain $\mathcal{T}(t)$, so hereafter we shall set it to zero for simplicity. The many-body Hamiltonian of the system is given by:

$$
\hat{H}_{\mathrm{S}}(t)=\sum_{i} \frac{\left(\hat{\vec{p}}_{i}+e \vec{A}\left(\vec{r}_{i}, t\right)\right)^{2}}{2 m}+\sum_{i<j} U\left(\hat{\overrightarrow{\vec{r}}_{i}}, \hat{\vec{r}}_{j}\right) .
$$

where we are working in the gauge where the scalar potential vanishes. $\hat{\vec{r}}_{i}$ and $\hat{\vec{p}}_{i}$ are the position and momentum of the $i$-th particle in the system, $\vec{A}$ is the external potential, $U$ is the interparticle interaction, $e$ and $m$ are the charge and mass of the particles. We shall be interested in the particle and current density operators, $\hat{n}(\vec{r})=\sum_{i} \delta\left(\vec{r}-\hat{\overrightarrow{r_{i}}}\right), \hat{\vec{j}}(\vec{r}, t)=\frac{1}{2} \sum_{i}\left\{\delta\left(\vec{r}-\hat{r}_{i}\right), \hat{\vec{v}}_{i}\left(\vec{r}_{i}, t\right)\right\}$, respectively. The canonical velocity operators $\hat{\vec{v}}_{i}(\vec{r}, t)$ are not just proportional to $\hat{\vec{p}}_{i}$, but equal to $\hat{\vec{v}}_{i}\left(\vec{r}_{i}, t\right)=\frac{1}{m}\left(\hat{p}_{i}+e \vec{A}\left(\hat{\vec{r}}_{i}, t\right)\right)$. An arbitrary property $O(\vec{r}, t)$ depending on the state of the system $\rho(t)$ will be computed as usual; if it represents an observable, it is calculated as $\langle\hat{O}(\vec{r}, t)\rangle_{t}=\operatorname{Tr}(\hat{O}(\vec{r}, t) \rho(t))$, where \langle\rangle$_{t}$ indicates a trace with respect to $\rho(t)$. We emphasize the fact that the time dependence of $\langle\hat{O}(\vec{r}, t)\rangle_{t}$ will stem both from the explicit time dependence of the operator $\hat{O}(\vec{r}, t)$ and from the evolution of $\rho(t)$ due to the ME (1). The property of interest can also be a non-observable arbitrary functional of $\rho(t)$, such as the purity $O(\vec{r}, t)=P_{t}=\operatorname{Tr}\left(\rho(t)^{2}\right)$.

Let us define some functions under fixed initial density matrix $\rho(0)$, interparticle potential $U\left(\vec{r}_{i}, \vec{r}_{j}\right)$, and memory kernel $\mathcal{K}\left(t, t^{\prime}\right)$ (see Fig. 1):

1. $\mathbb{F}: \vec{A}(\vec{r}, t) \rightarrow \rho(t)$ maps vector potentials to density matrices via the ME of eqn (1), with fixed $\rho(0), U\left(\vec{r}_{i}, \vec{r}_{j}\right)$, and $\mathcal{K}\left(t, t^{\prime}\right)$.

2. $\rrbracket_{O}: \rho(t) \rightarrow O(\vec{r}, t)[\rho(t)]$ maps density matrices to properties that are functionals of density matrices, such as observables.

We are in particular interested in the mappings $\rrbracket_{j}: \rho(t) \rightarrow$ $\langle\hat{\vec{j}} \vec{r}, t)\rangle$ and $I_{n, j}: \rho(t) \rightarrow\left(\langle\hat{n}(\vec{r})\rangle_{t},\langle\hat{\vec{j}}(\vec{r}, t)\rangle_{t}\right)$.

3. $\left.\mathbb{G}: \vec{A}(\vec{r}, t) \rightarrow\left(\langle\hat{n}(\vec{r})\rangle_{t},\langle\hat{\vec{j}} \vec{r}, t)\right\rangle_{t}\right)$ maps vector potentials to particle and current densities.

4. $\tilde{\mathbb{G}}: \vec{A}(\vec{r}, t) \rightarrow\langle\hat{\vec{j}}(\vec{r}, t)\rangle_{t}$ maps vector potentials to current densities.

Note that $\mathbb{G}=\mathbb{q}_{n, j} \mathbb{F}, \tilde{\mathbb{G}}=\rrbracket_{j} \mathbb{F}$. In one of our articles (Yuen-Zhou, Rodríguez-Rosario, and Aspuru-Guzik, ${ }^{51}$ hereafter denoted as YRA), we already defined the first three functions and showed in its Theorem 1 an analog of the RG and Vignale theorems, namely, that $\mathbb{G}$ is a one-to-one map. This statement is still correct, despite the challenges to it by ref. 64. An important consequence of this injection is that there exists the inverse map $\mathbb{G}^{-1}$, whose domain is equal to the image set of $\mathbb{G}$, which yields $\quad \rho(t)=\mathbb{F}^{-1}\left(\langle\hat{n}(\vec{r})\rangle_{t},\langle\hat{\vec{j}}(\vec{r}, t)\rangle_{t}\right) \quad$ and $\left.O(\vec{r}, t)=\rrbracket_{O} \mathbb{F} \mathbb{G}^{-1}\left(\langle\hat{n}(\vec{r})\rangle_{t},\langle\hat{\vec{j}} \vec{r}, t)\right\rangle_{t}\right)$. Importantly, we have emphasized that does not imply that every pair $\left(\langle\hat{n}(\vec{r})\rangle_{t},\langle\hat{\vec{j}}(\vec{r}, t)\rangle_{t}\right)$ can be inverted via $\mathbb{G}^{-1}$, for it might not be in the image set of $\mathbb{G}$ (the claim is that $\mathbb{G}$ is injective, ${ }^{51}$ not that it is bijective). Therefore, in principle, if both $\langle\hat{\vec{j}} \vec{r}, t)\rangle_{t}$ and $\langle\hat{n}(\vec{r})\rangle_{t}$ are sufficiently accurately reproduced via an alternative 


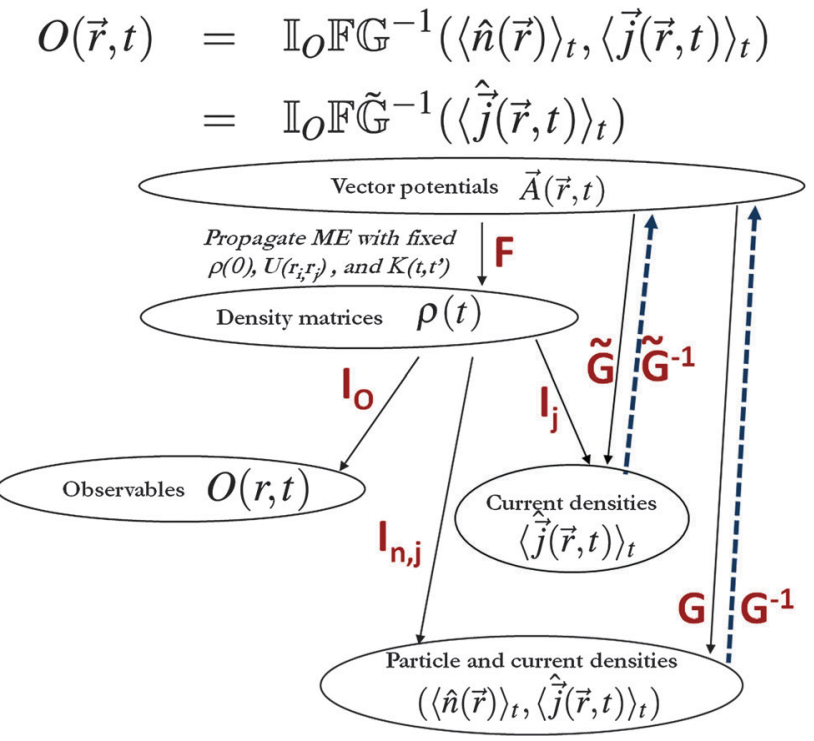

Fig. 1 Relevant mappings for TDCDFT. Given a fixed initial state $\rho(0)$, interparticle potential $U\left(\vec{r}_{i}, \vec{r}_{j}\right)$, and memory kernel $\mathcal{K}\left(t, t^{\prime}\right)$, $\mathbb{F}$ maps vector potentials $\vec{A}(\vec{r}, t)$ to density matrices $\rho(t)$ via the evolution of the ME. Once $\rho(t)$ is calculated, any properties of the system can be calculated from it, yielding the maps $\rrbracket_{O}$ from density matrices to arbitrary properties $\hat{O}(\vec{r}, t)$, and in particular, $\mathbb{n}_{n, j}$ to the particle and current density pair $\left(\langle\hat{n}(\vec{r})\rangle_{t},\langle\hat{\vec{j}}(\vec{r}, t)\rangle_{t}\right)$, and $\nabla_{j}$ to the current density $\langle\hat{\vec{j}}(\vec{r}, t)\rangle_{t}$ only. $\mathbb{G}=\mathbb{Q}_{n j} \mathbb{E}, \tilde{\mathbb{G}}=\mathbb{Q}_{j} \mathbb{E}$ are the composition maps from $\vec{A}(\vec{r}, t)$ directly to $\left(\langle\hat{n}(\vec{r})\rangle_{t},\langle\hat{\vec{j}}(\vec{r}, t)\rangle_{t}\right)$ and $\langle\hat{\vec{j}}(\vec{r}, t)\rangle_{t}$, respectively. In YRA, we have shown that $\mathbb{G}$ is one-to-one, yielding a well-defined inverse $\mathbb{G}^{-1}$ whose domain is the image set of $\mathbb{G}$. In the current article, we show that $\tilde{\mathbb{G}}$ is also one-to-one, and that the inverse $\mathbb{G}^{-1}$ exists, with its domain being the image set of $\tilde{\mathbb{G}}$.

way other than through the costly computation involved in $\mathbb{F}$, then the mapping $\mathbb{\square}_{0} \mathbb{F} \mathbb{G}^{-1}$ could be used to calculate any observables of the OQS as functionals of $\langle\hat{\vec{j}} \vec{r}, t)\rangle_{t}$ and $\langle\hat{n}(\vec{r})\rangle_{t}$. In practice, this would be achieved through a non-interacting KS system aimed at reproducing these two observables. $†$

\section{Remarks and discussions}

\section{A. $\tilde{\mathbb{G}}$ is injective (one-to-one) too}

We proceed to answer the following question: is $\tilde{\mathbb{G}}$ also injective? If so, analogous conclusions to the ones above associated with $\mathbb{G}$ would hold too. In YRA, we made an incorrect claim, implying that the answer is "no." Here, we correct our original statement.

Theorem. $\tilde{\mathbb{G}}$ is a one-to-one map.

Proof. The proof is similar to the one for Theorem 1 presented in YRA, provided some subtleties are addressed. Consider two systems: the original one, associated to $\rho(t)$, which evolves according to eqn (1). The auxiliary one, associated with $\rho^{\prime}(t)$, which evolves under the primed equation of motion,

$$
\frac{\mathrm{d} \rho^{\prime}(t)}{\mathrm{d} t}=-i\left[\hat{H}_{\mathrm{S}}^{\prime}(t), \rho^{\prime}\left(t^{\prime}\right)\right]+\int_{0}^{t} \mathrm{~d} t^{\prime} \mathcal{K}^{\prime}\left(t, t^{\prime}\right) \rho^{\prime}\left(t^{\prime}\right),
$$

† A conservation of the computational complexity is hidden in the practical construction of the composition map $\mathbb{}_{0} \mathbb{F} G^{-1}$, that is, the construction of observables as functionals of the current density. and yield the same current density as the original system:

$$
\left.\left\langle\hat{\vec{j}}^{\prime}(\vec{r}, t)\right\rangle_{t}^{\prime}=\langle\hat{\vec{j}} \vec{r}, t)\right\rangle_{t}
$$

Here, the "primed" Hamiltonian $\hat{H}_{\mathrm{S}}^{\prime}(t)$ is the same as eqn (2) except for the substitution $A \rightarrow A^{\prime}$. Assume throughout this proof that $\rho(0)=\rho^{\prime}(0), U=U^{\prime}$, and $\mathcal{K}\left(t, t^{\prime}\right)=\mathcal{K}^{\prime}\left(t, t^{\prime}\right)$ for all $t-t^{\prime} \geq 0$. Suppose that for all $\vec{r} \in \mathbb{R}^{3}$ and $t \geq 0$, the current densities are the same in both systems. We ask the question: under these circumstances, can $\vec{A} \neq \vec{A}^{\prime}$ for some value of $\vec{r}$ and $t$ ? The answer will be "no", hence proving the theorem.

The equations of motion for the particle and current densities of the original system are: ${ }^{8,48,51}$

$$
\left.\frac{\partial\langle\hat{n}(\vec{r})\rangle_{t}}{\partial t}=-\vec{\nabla} \cdot\langle\hat{\vec{j}} \vec{r}, t)\right\rangle_{t}+\lambda(\vec{r}, t)
$$

$$
\begin{aligned}
\frac{\partial\langle\hat{\vec{j}}(\vec{r}, t)\rangle_{t}}{\partial t}= & \frac{e\langle\hat{n}(\vec{r})\rangle_{t}}{m} \frac{\partial \vec{A}(\vec{r}, t)}{\partial t}-\frac{e\langle\hat{\vec{j}}(\vec{r}, t)\rangle_{t}}{m} \times(\vec{\nabla} \times \vec{A}(\vec{r}, t)) \\
& +\overrightarrow{\mathcal{D}}(\vec{r}, t)+\frac{\overrightarrow{\mathcal{F}}(\vec{r}, t)}{m}+\overrightarrow{\mathcal{G}}(\vec{r}, t) .
\end{aligned}
$$

Here, $\lambda(\vec{r}, t)=\operatorname{Tr}\left\{\hat{n}(\vec{r})\left(\int_{0}^{t} \mathrm{~d} t^{\prime} \mathcal{K}\left(t, t^{\prime}\right) \rho\left(t^{\prime}\right)\right)\right\}$ is the leakage term that restores the continuity equation for OQS. ${ }^{51}$ Whereas a closed system always satisfies the continuity equation, an OQS may not satisfy the continuity equation in general when the leakage term is nonzero. This can be regarded as a computational artifact of coarse-graining, and in fact, the current detected in an OQS experiment might not be the same as $\langle\hat{\vec{j}} \vec{r}, t)\rangle_{t}$, depending on the time resolution of the measurement apparatus. ${ }^{67-69}$ Nevertheless, for purposes of this article, we shall operationally keep referring to $\langle\hat{\vec{j}} \vec{r}, t)\rangle_{t}$ as the current density. The rest of the terms are given by $\overrightarrow{\mathcal{D}}(\vec{r}, t)=$ $-\frac{1}{4} \sum_{\alpha, \beta} \hat{\beta} \frac{\partial}{\partial \alpha}\left\langle\sum_{i}\left\{\hat{\vec{v}}_{i \alpha},\left\{\hat{\vec{v}}_{i \beta}, \delta\left(\vec{r}-\hat{\vec{r}}_{i}\right)\right\}\right\}\right\rangle$ for $\alpha, \beta=x, y, z$, $\overrightarrow{\mathcal{F}}(\vec{r}, t)=-\left\langle\sum_{i} \delta\left(\vec{r}-\hat{\vec{r}}_{i}\right) \sum_{j \neq i} \vec{\nabla}_{\vec{r}_{i}} U\left(\vec{r}_{i}-\vec{r}_{j}\right)\right\rangle, \quad$ and $\quad \overrightarrow{\mathcal{G}}(\vec{r}, t)=$ $\operatorname{Tr}\left\{\hat{\vec{j}}(\vec{r}, t)\left(\int_{0}^{t} \mathrm{~d} t^{\prime} \mathcal{K}\left(t, t^{\prime}\right) \rho\left(t^{\prime}\right)\right)\right\}$. Explanations of these terms can be found in YRA. Analogous primed relations hold for the auxiliary system.

Let us Taylor expand eqn (5) about $t=0$ as $O(\vec{r}, t)=$ $\sum_{k=0}^{\infty} O_{k}(\vec{r}) t^{k}$ with $\left.O_{k}(\vec{r}) \equiv \frac{1}{k !} \frac{\partial^{k} O(\vec{r}, t)}{\partial t^{k}}\right|_{t=0}$. Equating powers of $t$, we obtain,

$$
\ln _{l}(\vec{r})=-\vec{\nabla} \cdot \overrightarrow{j_{l-1}}(\vec{r})+\lambda_{l-1}(\vec{r}),
$$

after collecting $t^{l-1}$ terms. Note that eqn (4) can also be written as:

$$
\overrightarrow{j_{k}}(\vec{r})=\vec{j}_{k}^{\prime}(\vec{r}),
$$

for all $0 \leq k<\infty$. 
If $\mathcal{K}\left(t, t^{\prime}\right)$ is a smooth function of the time arguments, $\lambda_{k}$ depends at most on $\left.\frac{\partial^{k-1} \mathcal{K}\left(t, t^{\prime}\right)}{\partial t^{k-1}}\right|_{t^{\prime}=t}$ and $\frac{\partial^{k-1} \rho}{\partial t^{k-1}}$ (see YRA). $\vec{A}$ couples directly to the system and indirectly to the bath through the system. If there are any memory effects at time $t$ associated with $\vec{A}$, they must be due to $\vec{A}\left(\vec{r}, t^{\prime}\right)$ for $t^{\prime}$ strictly smaller than $t$. In other words, $\vec{A}$ couples to the system, the system couples to the bath, and only afterwards, in a second order process in the system-bath interaction can this information return to the system as memory through $\mathcal{K}\left(t, t^{\prime}\right)$. By these considerations, $\left.\frac{\partial^{k-1} \mathcal{K}\left(t, t^{\prime}\right)}{\partial t^{k-1}}\right|_{t=t^{\prime}}$ can depend at most on $\vec{A}_{k-2}(\vec{r})$. $\frac{\partial^{k-1} \rho}{\partial t^{k-1}}$ also depends at most on $\vec{A}_{k-2}(\vec{r})$ through the Hamiltonian in eqn (2). Therefore, in the case of $\mathcal{K}\left(t, t^{\prime}\right)$ smooth as a function of $t$ and $t^{\prime}, \lambda_{k}(\vec{r})$ depends at most on $\vec{A}_{k-2}(\vec{r})$. A proof using formal arguments derived from projector operator methods yield the same conclusion. ${ }^{70,71}$ For the important Markovian limit where $\mathcal{K}\left(t, t^{\prime}\right)$ is not a smooth function and $\mathcal{K}\left(t, t^{\prime}\right)=$ $\mathcal{L}(t) \delta\left(t-t^{\prime}\right)$ for some superoperator $\mathcal{L}$. By the same arguments as above, $\frac{\partial^{k} \mathcal{L}}{\partial t^{k}}$ should depend at most on $\vec{A}_{k-1}(\vec{r})$ (the most common situation is when $\mathcal{L}$ is constant in time). $\lambda_{k}$ can also depend on $\frac{\partial^{k} \rho}{\partial t^{k}}$, which depends at most on $\vec{A}_{k-1}(\vec{r})$. Therefore, the summary is that in either case, $\lambda_{k}$ depends at most on $\vec{A}_{k-1}(\vec{r})$.

The next step in the proof is an induction. Consider the strong inductive hypothesis:

$$
\vec{A}_{k}(\vec{r})=\vec{A}_{k}^{\prime}(\vec{r})
$$

for all $0 \leq k<l$ where $l \geq 1$. Two statements follow:

(a) Eqn (8) together with the arguments in the previous paragraph imply that: $\neq$

$$
n_{l}(\vec{r})=n_{l}^{\prime}(\vec{r})
$$

which means that at each step, we prove that the particle densities in both systems must be the same.

(b) Let us Taylor expand eqn (6) and its primed version, and use eqn (8) and (10) to collect terms associated with $t^{l}$ :

$$
\begin{aligned}
e(l+1) n_{0}(\vec{r}) \Delta \vec{A}_{l+1}(\vec{r})= & -e \sum_{k=0}^{l-1}(k+1) n_{l-k}(\vec{r}) \Delta \vec{A}_{k+1}(\vec{r}) \\
& +e \sum_{k=0}^{l} \vec{j}_{l-k}(\vec{r}) \times\left(\vec{\nabla} \times \Delta \vec{A}_{k}(\vec{r})\right) \\
& +\left(m \overrightarrow{\mathcal{D}}_{l}(\vec{r})+\overrightarrow{\mathcal{F}}_{l}(\vec{r})+m \overrightarrow{\mathcal{G}}_{l}(\vec{r})\right) \\
& -\left(m \overrightarrow{\mathcal{D}}_{l}^{\prime}(\vec{r})+\overrightarrow{\mathcal{F}}_{l}^{\prime}(\vec{r})+m \overrightarrow{\mathcal{G}}_{l}^{\prime}(\vec{r})\right),
\end{aligned}
$$

$\ddagger$ In fact, to guarantee this first statement, we only need $\vec{A}_{k}(\vec{r})=\vec{A}_{k}^{\prime}(\vec{r})$ for all $0 \leq$ $k \leq l-2$, because eqn (7) depends on $\lambda_{l-1}(\vec{r})$ which, as we have just concluded, depends at most on $\vec{A}_{l-2}(\vec{r})$. where $\Delta A=A^{\prime}-A$. Eqn (11) is the same as eqn (10) in YRA. The same immediate consequence of that investigation is obtained, namely, that the right hand of the equation contains $\Delta \vec{A}_{l}$ as its highest order derivative (we refer the reader to YRA for a detailed derivation of this conclusion). Hence, due to the hypothesis in eqn (9), the right hand side vanishes identically to zero.

We now prove eqn (9) for the basis case for the induction, $l=1$. Since the initial states in both systems are the same, $n_{0}(\vec{r})=n_{0}^{\prime}(\vec{r})=\langle\hat{n}(\vec{r})\rangle_{0}$. Eqn (8) holds for any $k$, in particular for $k=0$. This yields:

$$
\Delta \vec{A}_{0}(\vec{r})=\frac{\operatorname{Tr}\left(\left(\rho(0)-\rho^{\prime}(0)\right)\left(\sum_{i}\left\{\vec{p}_{i}, \delta\left(\vec{r}-\vec{r}_{i}\right)\right\}\right)\right)}{2 e\langle\hat{n}(\vec{r})\rangle_{0}}=0 .
$$

Eqn (11) also holds for $l=0$, and gives $\Delta \vec{A}_{1}(\vec{r})=0$, proving the basis case. By induction, $\Delta \vec{A}_{k}(\vec{r})=0$ for all $0 \leq k<\infty$, so $\vec{A}(\vec{r}, t)=\vec{A}^{\prime}(\vec{r}, t)$ for all $t$ in the domain of convergence of all the Taylor expansions. The proof can be repeated Taylor expanding about different times until they cover the entire $t \geq 0$ domain.

In passing, note that eqn (4) together with the rest of the hypotheses imply eqn (10), which means $\langle\hat{n}(\vec{r})\rangle_{t}=\langle\hat{n}(\vec{r})\rangle_{t}^{\prime}$ once the induction proof is over. In YRA, we had regarded the latter as an additional hypothesis, but here we realize that such assumption is a consequence of the other hypotheses of the current theorem.

Discussion. Theorem 1 in YRA and the Theorem in this article altogether claim that both $\mathbb{G}$ and $\tilde{\mathbb{G}}$ are injective maps. Hence, it is only necessary to reproduce $\langle\hat{\vec{j}}(\vec{r}, t)\rangle_{t}$ in the KS system because then, the properties of the original system can be expressed as $\left.O(\vec{r}, t)=\rrbracket_{O} \mathbb{F} \tilde{\mathbb{G}}^{-1}(\langle\hat{\vec{j}} \vec{r}, t)\rangle_{t}\right)$. In YRA, we had criticized the KS scheme presented in ref. 48 and 49 based on the fact that, in general, it is not possible to reproduce $\langle\hat{n}(\vec{r})\rangle_{t}$ and $\langle\hat{\vec{j}}(\vec{r}, t)\rangle_{t}$ simultaneously using a KS system which is restricted to dissipate with the same bath operators as the original system. The latter is indeed the case for microscopically derived MEs of the Lindblad form in the weak system-bath coupling regime, where the dissipation operators are in general non-local, yielding finite leakage terms $\lambda(\vec{r}, t)$ (see eqn (5)). ${ }^{51,67-69}$ However, this fact is immaterial for the establishment of a KS scheme, as only $\langle\hat{\vec{j}} \vec{r}, t)\rangle_{t}$ needs to be the same in both original and KS system, irrespective of $\langle\hat{n}(\vec{r})\rangle_{t}$. This subtlety was not appreciated by YRA or by others, such as in ref. 49 , where the authors state "... these are the only two physical quantities that the KS system needs to reproduce" (first paragraph, page 7 in

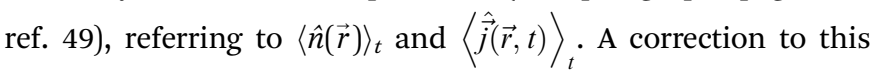
statement by the same authors is later reported in ref. 64 and 65 .

It is important, however, to stress that none of the KS schemes we suggest in YRA, which are intended to reproduce both $\langle\hat{n}(\vec{r})\rangle_{t}$ and $\langle\hat{\vec{j}}(\vec{r}, t)\rangle_{t}$ via alternative methods, are affected by the theorem in the present article, and remain valid as KS 
schemes for TDCDFT for OQS, as the reproduction of both $\langle\hat{n}(\vec{r})\rangle_{t}$ and $\langle\hat{\vec{j}}(\vec{r}, t)\rangle_{t}$ in the KS system implies at least the reproduction of $\langle\hat{\vec{j}} \vec{r}, t)\rangle_{t}$. In other words, our YRA scheme was redundant, but correct. In particular, our central result (Theorem 3 in YRA) of using a CDS as a KS scheme holds, and its variant in the context of TDDFT $^{53}$ has been correctly proposed, together with a practical Markovian bath functional. The TDDFT quantum Drude approach for dissipation due to Neuhauser ${ }^{50}$ can also be regarded as a mapping from an original OQS to a CDS KS scheme.

Also, restating the ideas from last section, let us illustrate how $\mathbb{G}$ is injective, but not necessarily bijective. Simply, take a $\langle\vec{j}(\vec{r}, t)\rangle_{t}$ given by some $\rho(t)$, which in turn, is produced by an $\vec{A}(\vec{r}, t)$, and consider all the pairs $\left(\langle\hat{n}(\vec{r})\rangle_{t},\langle\hat{\vec{j}}(\vec{r}, t)\rangle_{t}\right)$ for which $\langle\hat{n}(\vec{r})\rangle_{t}$ does not satisfy eqn (5). These pairs are not in the image set of $\mathbb{G}$ and hence cannot be inverted by $\mathbb{G}^{-1}$ : no $\vec{A}$ produces them via eqn (1) for fixed $\rho(0), U\left(\vec{r}_{i}, \vec{r}_{j}\right)$, and $\mathcal{K}\left(t, t^{\prime}\right)$. Hence, the domain of $\mathbb{G}^{-1}$ is restricted to the image set of $\mathbb{G}$, as explained before and in ref. 51 . Contrary to previous criticism, ${ }^{64}$ this restriction allows for the well-defined inversion of observables as functionals of $\left(\langle\hat{n}(\vec{r})\rangle_{t},\langle\hat{\vec{j}}(\vec{r}, t)\rangle_{t}\right)$ via $O(\vec{r}, t)=\mathbb{\square}_{O} \mathbb{E} \mathbb{G}^{-1}\left(\langle\hat{n}(\vec{r})\rangle_{t},\langle\hat{\vec{j}}(\vec{r}, t)\rangle_{t}\right)$.

\section{B. No inconsistency in the mapping from the original open quantum system to the Kohn-Sham closed driven system}

To aid in the following arguments, we reproduce Theorem 3 from YRA:

"Theorem 3. (A,C Kohn Sham scheme) There exists an auxiliary closed KS system which starting in the state $\rho_{\mathrm{KS}}(0)$ evolves unitarily under a KS Hamiltonian $\hat{H}_{\mathrm{KS}}(t)$ :

$$
\hat{H}_{\mathrm{KS}}(t)=\sum_{i} \frac{\left(\hat{\vec{p}}_{i}+e\left(\vec{A}_{\mathrm{KS}}\left(\vec{r}_{i}, t\right)+\vec{C}\left(\vec{r}_{i}, t\right)\right)\right)^{2}}{2 m}+\sum_{i<j} U\left(\vec{r}_{i}, \vec{r}_{j}\right)
$$

producing particle and filtered current densities that are related to the particle and current densities of the original many body interacting OQS governed by (1) by:

$$
\begin{aligned}
\langle\hat{n}(\vec{r})\rangle_{\mathrm{KS}, t} & =\langle\hat{n}(\vec{r})\rangle_{t} \\
\langle\hat{\vec{j}} & \left.(\overrightarrow{i l t}, t)\rangle_{\mathrm{KS}, t}=\langle\hat{\vec{j}} \vec{r}, t)\right\rangle_{t}
\end{aligned}
$$

where we have defined the filtered current operator as $\hat{\overrightarrow{\vec{j}}}_{\text {filt }}(\vec{r}, t)=\sum_{i} \frac{1}{2}\left\{\hat{\vec{v}}_{i, \text { filt }}(\vec{r}, t), \delta\left(\vec{r}-\hat{\vec{r}}_{i}\right)\right\}$ with the filtered velocity operator given by $\hat{\vec{v}}_{i \text { filt }}\left(\vec{r}_{i}, t\right)=\frac{\hat{\vec{p}}_{i}+e \vec{A}_{\mathrm{KS}}\left(\hat{\vec{r}}_{i}, t\right)}{m}$. Additionally, we denote the expectation values in the KS system in the usual form $\langle\hat{O}(\vec{r}, t)\rangle_{\mathrm{KS}, t}=\operatorname{Tr}\left(\hat{O}(\vec{r}, t) \rho_{\mathrm{KS}}(t)\right)$. We shall call $\vec{A}_{\mathrm{KS}}(\vec{r}, t)$ the KS vector potential, and $\vec{C}(\vec{r}, t)$ the leakage potential."

Discussion. Theorem 3 in YRA establishes the possibility to reproduce both $\langle\hat{n}(\vec{r})\rangle_{t}$ and $\langle\hat{\vec{j}}(\vec{r}, t)\rangle_{t}$ as expectation values of appropriately defined operators using a $\operatorname{CDS}\left(\mathcal{K}\left(t, t^{\prime}\right)=0\right)$. Clearly, due to the leakage term $\lambda(\vec{r}, t)$ which restores continuity in eqn (5), it is in general impossible to reproduce both $\langle\hat{n}(\vec{r})\rangle_{t}$ and $\langle\hat{\vec{j}} \vec{r}, t)\rangle_{t}$ of an OQS with a CDS. Therefore, we show that by appropriately defining a filtered current operator, its expectation value on the CDS can reproduce $\langle\hat{\vec{j}}(\vec{r}, t)\rangle_{t}$ of the original OQS. The particle density operator is defined in the usual way in both original and KS DCS systems. Contrary to criticisms, ${ }^{64}$ we are not trying to reproduce the full many-body dynamics of the original OQS with the closed system, but only trying to reproduce $\langle\hat{n}(\vec{r})\rangle_{t}$ and $\langle\hat{\vec{j}}(\vec{r}, t)\rangle_{t}$ via a well-defined procedure. The reproduction of these two observables and not the full dynamics is what most TD(C)DFT schemes are about. Since the OQS under consideration exchange energy with an environment, but do not lose particles as time evolves, it is quite conceivable that there exists a CDS which mimics the particle and current density profiles of the OQS by pumping energy in and out of the system via the time-dependent external potentials. Obviously, there will be properties which differ in both systems, such as the purity, $P_{t}=\operatorname{Tr}\left(\rho(t)^{2}\right) \neq \operatorname{Tr}\left(\rho_{\mathrm{KS}}(t)^{2}\right)=$ $P_{t, \mathrm{KS}}$. Whereas $P_{t}$ changes in time as it becomes entangled with the bath, $P_{t, \mathrm{KS}}$ stays constant throughout the unitary evolution. Nevertheless, there is no flaw in the logic of the formalism we have presented: even $P_{t}$ could in principle be calculated as a functional of the current density, which is obtained through the KS system, $\left.P_{t}=\rrbracket_{O} \mathbb{E} \tilde{\mathbb{G}}^{-1}(\langle\hat{\vec{j}} \vec{r}, t)\rangle_{t}\right)$, where $O=P_{t}$. This functional, however, might not be trivial to find in practice.

Note that $\vec{A}_{\mathrm{KS}}$ and $\vec{C}$ are not unique (in fact, they do not need to be for Theorem 3 in YRA to be true). As explained in the proof in YRA, the continuity equation of the unitary evolution of the KS system is given by:

$$
\frac{\partial\langle\hat{n}(\vec{r})\rangle_{\mathrm{KS}, t}}{\partial t}=-\vec{\nabla} \cdot\left\langle\hat{\vec{j}}_{\mathrm{filt}}(\vec{r}, t)\right\rangle_{\mathrm{KS}, t}-\vec{\nabla} \cdot\left(\frac{e \vec{C}(\hat{\vec{r}}, t)}{m}\langle\hat{n}(\vec{r})\rangle_{\mathrm{KS}, t}\right) .
$$

Let us define the variable $\vec{G}(\vec{r}, t) \equiv-\frac{e \vec{C}(\hat{\vec{r}}, t)}{m}\langle\hat{n}(\vec{r})\rangle_{\mathrm{KS}, t}$. By virtue of the Helmholtz decomposition, it is always possible to write $\vec{G}=\vec{G}_{\perp}+\vec{G}_{\|}$, where $\perp$ and $\|$ denote the transverse and longitudinal components of the vector field, defined by the relations $\vec{\nabla} \cdot \vec{G}_{\perp}=0$ and $\vec{\nabla} \times \vec{G}_{\|}=0$. The divergenceless condition of $\vec{G}_{\perp}$ implies that it may be expressed as the curl of a vector field $\vec{g}(\vec{r}, t), \vec{G} \perp(\vec{r}, t)=\vec{\nabla} \times \vec{g}(\vec{r}, t)$. Similarly, the curlless condition of $\vec{G}_{\|}$means that there is a scalar field $h(\vec{r}, t)$ such that $\vec{G}_{\|}(\vec{r}, t)=\vec{\nabla} h(\vec{r}, t)$. Imposing the constraint due to eqn (15), one can see that whereas $\vec{g}$ can be an arbitrary field, $\vec{h}$ must satisfy the Poisson equation,

$$
\nabla^{2} h=\frac{\partial\langle\hat{n}(\vec{r})\rangle_{\mathrm{KS}, t}}{\partial t}+\vec{\nabla} \cdot\left\langle\hat{\vec{j}}_{\mathrm{filt}}(\vec{r}, t)\right\rangle_{\mathrm{KS}, t}
$$

which is uniquely determined given the boundary condition at some surface, for instance, at $|\vec{r}| \rightarrow \infty$. Clearly, the 
arbitrariness of $\vec{G}_{\perp}(\vec{r}, t)$ via $\vec{g}(\vec{r}, t)$ as well as that of $\vec{G}_{\|}(\vec{r}, t)$ via the (arbitrary) boundary condition of $h$ in turn gives quite a bit of freedom in the determination of $\vec{C}(\vec{r}, t)$, as DADV correctly note; $\vec{C}(\vec{r}, t)$ is not uniquely defined. For instance, a possible $\vec{C}(\vec{r}, t)$ is,

$$
\vec{C}(\vec{r}, t)=-\hat{x} \frac{m}{e\langle\hat{n}(\vec{r})\rangle_{t}} \int_{0}^{x} \mathrm{~d} x^{\prime}\left(\frac{\partial\left\langle\hat{n}\left(\vec{r}^{\prime}\right)\right\rangle_{t}}{\partial t}+\vec{\nabla} \cdot\left\langle\hat{\vec{j}}\left(\vec{r}^{\prime}, t\right)\right\rangle_{t}\right),
$$

but this is not unique. Here, the vector field points in the $\hat{x}$ direction, $\vec{r}=(x, y, z)$, and the lower limit of the integral is at $(0, y, z)$. The arbitrariness of $\vec{C}(\vec{r}, t)$, however, is immaterial: so long as it satisfies eqn (15), it will subtract the correct amount of leakage current from the total KS current, so that the second constraint in eqn (14) is fullfilled.

The proof for the theorem in discussion (see YRA) explains how to construct $\vec{A}_{\mathrm{Ks}}$ from $\vec{C}$. Furthermore, we can construct $\vec{A}_{\mathrm{KS}}^{\prime \prime}$ from another $\vec{C}^{\prime \prime}$ which also guarantees eqn (14). In general, $\vec{C}+\vec{A}_{\mathrm{KS}} \neq \vec{C}^{\prime \prime}+\vec{A}_{\mathrm{KS}}^{\prime \prime}$. Hence, the "total" KS vector potential $\vec{C}+\vec{A}_{\mathrm{KS}}$ is not unique, and two different total KS vector potentials yield the same particle and filtered current densities, $\langle\hat{n}(\vec{r})\rangle_{\mathrm{KS}, t}$ and $\left.\langle\hat{\vec{j}} \vec{r}, t)\right\rangle_{\mathrm{KS}, t}$, respectively. As opposed to what is claimed in ref. 64 , this fact is not in contradiction with Theorem 1. In general, the total current densities $\langle\hat{\vec{j}}(\vec{r}, t)\rangle_{\mathrm{KS}, t}$ due to $\vec{C}+\vec{A}_{\mathrm{KS}}$ and $\vec{C}^{\prime \prime}+\vec{A}_{\mathrm{KS}}^{\prime \prime}$ will be different, in consistency with Theorem 1, which reduces to Vignale's theorem as the KS system is taken to be closed. ${ }^{8}$ Yet, $\left.\langle\hat{\vec{j}} \vec{r}, t)\right\rangle_{\mathrm{KS}, t}$ can be regarded simply as computational means to obtain the particle and filtered current densities, $\langle\hat{n}(\vec{r})\rangle_{\mathrm{KS}, t}$ and $\left\langle\hat{\vec{j}}_{\text {filt }}(\vec{r}, t)\right\rangle_{\mathrm{KS}, t}$, which are by construction, the same using either KS vector potential, and supposed to correspond to the actual OQS observables, as the constraint in eqn (14) indicates.

We reiterate that the KS scheme conceived here is in no contradiction with the theorem in the current article, since the former intends to extract both $\langle\hat{n}(\vec{r})\rangle_{t}$ and $\left.\langle\hat{\vec{j}} \vec{r}, t)\right\rangle_{t}$ of the original OQS, whereas the latter requires that only the extraction of $\langle\hat{\vec{j}}(\vec{r}, t)\rangle_{t}$ is necessary. The correct extraction of $\langle\hat{n}(\vec{r})\rangle_{t}$ is, as argued here, redundant, but by no means incorrect.

\section{Positivity of the Kossakowski-Lindblad master equation irrespective of time-dependence of Hamiltonian and dissipation operators}

A quantum $\mathrm{ME}$ is the generic denomination of an equation of motion for the density matrix of an OQS. A systematic approach to derive a $\mathrm{ME}$ from a microscopic model is given by the Nakajima-Zwanzig projection operator method. ${ }^{71-74}$ Alternatively, phenomenological approaches are also often advocated.

A popular form of Markovian ME which is often used in the literature is the generalized Kossakowski-Lindblad (KL) equation: $:^{75-77}$

$$
\frac{\partial \rho}{\partial t}=\mathcal{L}(\rho) \equiv-i[\hat{H}, \rho]+\sum_{i=1}^{N}\left(-\frac{1}{2} \hat{L}_{i}^{\dagger} \hat{L}_{i} \rho-\frac{1}{2} \rho \hat{L}_{i}^{\dagger} \hat{L}_{i}+\hat{L}_{i} \rho \hat{L}_{i}^{\dagger}\right),
$$

where both $\hat{H}$ and $\hat{L}_{i}$ can be time-dependent. This ME is known to preserve the positivity and trace of the density matrix under its evolution, irrespective of the time-dependence of $\hat{H}$ and $\hat{L}_{i}$. To verify this, we expand $\rho(t+\Delta t)$ for small $\Delta t$ :

$$
\begin{aligned}
\rho(t+\Delta t) & =\rho(t)+\frac{\partial \rho}{\partial t} \Delta t+O\left(\Delta t^{2}\right) \\
& \approx \sum_{i=0}^{N} M_{i}(\Delta t) \rho(t) M_{i}(\Delta t)^{\dagger} .
\end{aligned}
$$

Using eqn (18), we can derive the so-called Kraus operators $M_{i}(\Delta t)$, which are:

$$
M_{i}(\Delta t)=\hat{L}_{i} \sqrt{\Delta t}
$$

for $i>0$, and

$$
M_{0}(\Delta t)=I+\left(-i \hat{H}-\frac{1}{2} \sum_{i=1}^{N} \hat{L}_{i}^{\dagger} \hat{L}_{i}\right) \Delta t
$$

An important property of the Kraus operators is that they satisfy:

$$
\sum_{i=0}^{N} M_{i}(\Delta t)^{\dagger} M_{i}(\Delta t)=I
$$

as can be easily checked from eqn (20) and (21). Notice that, in general, $M_{i}(\Delta t)$ are time dependent if $\hat{L}_{i}$ are too.

Let us write $\rho(t)=\sum_{i} p_{j}\left|\xi_{j}(t)\right\rangle\left\langle\xi_{j}(t)\right|$, where $\left\{\left|\xi_{i}(t)\right\rangle\right\}$ is the basis that diagonalizes $\rho(t)$ with $p_{j} \geq 0$ for all $j$. Notice that up to $O\left(\Delta t^{2}\right)$ :

$$
\rho(t+\Delta t)=\sum_{j} \sum_{i=0}^{N} p_{j}\left(M_{i}(\Delta t)\left|\xi_{j}(t)\right\rangle\right)\left(\left\langle\xi_{j}(t)\right| M_{i}(\Delta t)^{\dagger}\right)
$$

which evidences that $\rho(t+\Delta t)$ is positive semidefinite if $\rho(t)$ is. The preservation of the trace can also be readily shown using eqn (22) and the cyclic invariance of the trace:

$$
\operatorname{Tr}(\rho(t+\Delta t))=\operatorname{Tr}\left(\sum_{i=0}^{N} M_{i}(\Delta t) \rho(t) M_{i}(\Delta t)^{\dagger}\right)=\operatorname{Tr}(\rho(t)) .
$$

The proofs above are a "backwards" adaptation of the standard textbook derivation of the KL equation as the generator of a completely positive map. See, for instance, the textbook. $^{78}$

For completeness, we now introduce the concept of a semigroup. Consider the integrated form of the equation of motion for $\rho(t)$ in the form of a dynamical map, $\rho(t)=\Phi_{t, 0} \rho(0)$, where $\Phi_{t, 0}$ is a dynamical map that propagates the density matrix from 0 to the final time $t$. The semigroup property can be expressed as the following identity for the composition map: ${ }^{71}$

$$
\Phi_{s, 0} \Phi_{t, 0}=\Phi_{s+t, 0}
$$


Discussion. On the one hand, as it is stated in ref. 49, the semigroup property will in general not be satisfied in general for time dependent $\hat{H}$ or $\hat{L}_{i}$. For instance, these operators at times belonging to the interval $[t, t+s]$ can differ significantly from their values at $[0, s]$. On the other hand, for the case where both types of operators are time-independent, it has been shown $^{76}$ that $\mathcal{L}$ is the most general form of the generator of a quantum dynamical semigroup, with the dynamical map being the exponential map: $\Phi_{s, 0}=e^{\mathcal{L} s}$. However, contrary to what ${ }^{49}$ and DADV claim, neither positivity nor trace-preservation depend on the time-dependence of $\hat{H}$ or $\hat{L}_{i}$, as we have explicitly shown here.

\section{No proof yet for the Runge-Gross theorem analog for individual trajectories in the stochastic Schrödinger equation}

It is well known that if $\hat{H}$ does not depend on the state of the system, eqn (18) can be "unraveled" as the evolution of an ensemble of Stochastic Schrödinger Equations (SSE) which reconstructs the density matrix upon appropriate manipulation of the calculated trajectories. ${ }^{60,79}$ Hence, for this situation, the SSE yields the same density matrix dynamics as the KL-ME in eqn (18). There are, however, several interesting features of the SSE that make it attractive compared to the ME, such as its lower numerical cost ${ }^{80}$ as well as the novel conceptual insights it provides based on measurement theory. ${ }^{81}$ In any case, the theorems in the work by Burke and coworkers ${ }^{46,47}$ and by us in YRA and, ${ }^{53}$ all of which are derived from the ME approach, can be implemented in the SSE KS scheme. An important point is that the KS vector or scalar potential would need to be calculated as a functional of the ensemble-averaged $\langle\hat{\vec{j}} \vec{r}, t)\rangle$ or $\langle\hat{n}(\vec{r}, t)\rangle$, respectively. Hence, one could devise a parallel algorithm where all the trajectories in the ensemble are propagated simultaneously, and at each time-step, the ensemble-averaged $\langle\hat{\vec{j}} \vec{r}, t)\rangle$ or $\langle\hat{n}(\vec{r}, t)\rangle$ computed, from which the KS potentials would be calculated as functionals. However, this is not what ${ }^{49}$ propose. Instead, they suggest the evolution of ensembles of wavefunctions evolving under different stochastic KS potentials which depend on each individual trajectory (see ref. 48, 49 and 64 for their general discussion, and ref. 66 for their TDDFT implementation). Unfortunately, the one-to-one mapping from single trajectory stochastic external potentials to the corresponding stochastic particle and current densities has not been proven. Hence, insofar this proof does not exist, the KS scheme in ref. 48 and 49 lacks theoretical foundation.

Discussion. The authors of ref. 48 and 49 claim that no closed equation of motion for the density matrix can be derived in this last situation (see paragraph before Section C in ref. 49). We agree with them on this point. As an illustration, consider the term $\langle\vec{A}(\vec{r}, t) \hat{n}\rangle$. If $\vec{A}(\vec{r}, t)$ is constant throughout the ensemble of trajectories, then we can write, $\langle\vec{A}(\vec{r}, t) \hat{n}(\vec{r})\rangle=\vec{A}(\vec{r}, t)\langle\hat{n}(\vec{r})\rangle$ and $\frac{\partial\langle\hat{\vec{j}}(\vec{r}, t)\rangle_{t}}{\partial t}=\frac{e\langle\hat{n}(\vec{r})\rangle_{t}}{m} \frac{\partial \vec{A}(\vec{r}, t)}{\partial t}+\cdots$, just as in eqn (6), which was derived from the ME. However, if $\vec{A}(\vec{r}, t)$ depends on each stochastic trajectory, $\langle\vec{A}(\vec{r}, t) \hat{n}(\vec{r})\rangle \neq\langle\vec{A}(\vec{r}, t)\rangle\langle\hat{n}(\vec{r})\rangle$ in general, and a closed equation of motion for the current density cannot be derived. Hence, the equations of motion for the current displayed in ref. 48 and 49 do not hold for this case. They hold for the case where the same $\vec{A}(\vec{r}, t)$ acts on each member of the ensemble, which coincides with the domain where the SSE is equivalent to the ME. Another verification is that our equation of motion for the current ${ }^{51,53}$ reduces to theirs in the limit where the memory kernel is of the KL form. A proof relying on these equations of motion cannot be a proof for the Runge-Gross analog for individual trajectories in the Stochastic Schrödinger Equation. At this point, we are not in the position to claim that there is no such Runge-Gross analog, but so far, if such proof exists, it has not been published.

It seems that authors in ref. 48 and 49 are aware of this issue but they still advocate their method. According to them, having a single $\vec{A}_{\mathrm{KS}}$ for the entire ensemble "requires that the exchange-correlation vector potential included also the statistical correlations of the direct Coulomb interaction at different points in space" (see discussion around eqn (43) in ref. 49). It is difficult for us to understand what this statement precisely means, and why the problem would be solved with the method they suggest. Also in the cited page, the authors worry about lack of positivity of the density matrix by using only one timedependent $\vec{A}_{\mathrm{Ks}}$ potential for all the trajectories. As shown in the previous section, this preoccupation is unfounded.

Having pointed out these subtleties, it might be the case that even if the KS scheme of ref. 48 and 49 is not rigorous, it is a pragmatic and useful approximate computational tool. However, it would be interesting to obtain further justification of the preference for their method as opposed to the more rigorous one described above.

We emphasize that at this point we are not claiming there is no Runge-Gross analogue theorem for the correspondence between stochastic densities and stochastic potentials for each trajectory. There simply has not been a published proof for it yet. Clearly, regardless of whether this proof appears, our ME approaches remain valid.

\section{E. Deficiencies of arbitrary master equation approaches}

A ME can be systematically derived from a microscopic model under the Nakajima-Zwanzing projector operator method. ${ }^{71,74}$ This approach is by far the most general one for OQS, and applies both to Markovian and non-Markovian dynamics, but might suffer from some problems due to the approximations involved in their derivations, such as the lack of positivity. In particular, it has been shown that this deficiency has a physical origin, as most of the MEs are only compatible with certain class of initial states, or are only applicable in some parameter range. ${ }^{82-84}$ An alternative approach is the restriction of the equations of motion to satisfy some minimal requirements such as positivity and trace preservation. The KL equation is an example of the latter approach, ${ }^{85,86}$ but non-Markovian analogues also exist. ${ }^{87}$ Even in these cases, it is difficult to construct MEs which satisfy all the desirable requirements of an OQS evolution. For instance, Kohen and Tannor have shown that no 
Markovian ME simultaneously satisfies the desired triad of positivity, translational invariance, and asymptotic approach to thermal equilibrium. ${ }^{88}$

Discussion. DADV criticize our study of generalized $\mathrm{ME}$ because they might not maintain positivity in general. As implied above, we are not aware of a ME which fullfills all the desirable physical requirements of an OQS evolution. This is true even in the context of the KL equation, which although does not violate positivity, is not translationally invariant, yielding different dissipative dynamics under translation of the coordinate system, a phenomenon which is not physical. ${ }^{88}$ Another example is the class of non-secular Redfield equations, which violate positivity under certain regimes, but which have been extensively used in the chemical physics community because they can be transparently derived from a microscopic model. ${ }^{89-92}$

Even if ME suffer from unavoidable deficiencies, they are one of the most promising ways to study OQS coupled to large environments. Hence, they are an invaluable theoretical tool and their connection with TDDFT should pave the way for the description of a wide variety of interesting OQS.

\section{Conclusions}

In this article, we have addressed several formal issues concerning our previous work ${ }^{51,53}$ and that of others ${ }^{48,49,66}$ in the topic TDDFT for OQS. In summary, we have found an incorrect speculation we had previously advocated in ref. 51, namely, that the map $\mathbb{G}$ from vector potentials to current densities is not injective, when in reality it is, as the theorem in this article establishes. Fortunately, the theorem in this article does not render any of the theorems in YRA as invalid, and therefore, all the suggested KS schemes proposed in our investigation remain rigorously applicable. In particular, we have clarified that using a KS CDS to compute the particle and current densities of the original OQS is well founded. The intuition behind this result is that the OQS under consideration exchange energy with an environment, but not particles. Time-dependent potentials driving closed systems also pump energy in and out of the system. Since only simple variables of the OQS such as particle and current densities profiles are supposed to be mimicked by the KS system, it is not surprising that we can achieve this with a CDS. Obviously, there are many properties that will not be the same in both systems, but that is expected from the TD(C)DFT theorems, as they only claim that those properties may be calculated as the functionals of particle and current densities, which in principle, are extracted correctly from the KS system. We also argued that uniqueness of several KS potentials is irrelevant in our scheme.

Contrary some recent views in the literature, we have shown that the KL equation maintains positivity even in the case of time-dependent Hamiltonians or dissipation operators. We also carefully compared the ME approach against the SSE formalism advocated by ref. 48,49 and 66 . If the potential does not depend on the stochastic wavefunction itself, then the SSE is equivalent to the KL-ME. However, the cited works place an important emphasis to the case where the potential depends on the stochastic wavefunction, yielding an ensemble of different potentials that govern each of the stochastic trajectories. Under such circumstances, just as those authors claim, no closed equation of motion for the observables can be derived. Yet, since their TDDFT proof in ref. 48 depends on such equations of motion, they must limit the practice of their theory to the same realm as the one of the MEs, unless there is a pragmatic reason to skip the rigor they have provided themselves. An alternative is to attempt to prove a Runge-Gross analog for stochastic trajectories, which to our knowledge, has not been published so far. MEs may violate certain desirable physical properties, but they are still very important constructs for the study of OQS. As far as our work is concerned, our mappings of TD(C)DFT based on MEs provide a rigorous framework to develop computational tools for the study of OQS. The development of dissipative functionals is at its infancy, ${ }^{50,53,55}$ but we foresee interesting work on this realm.

In summary, in this article, we have attempted to clarify several formal aspects of TDDFT for OQS, emphasizing on certain mappings and KS schemes which have caused some confusion in the recent literature. We have also compared the different schemes based on ME and SSE. We hope that the results and arguments presented here will aid the further development of the field.

\section{Acknowledgements}

D. G. Tempel and S. Boixo have been instrumental for the elucidation of several of the arguments presented in here. E. K. U. Gross, K. Burke, and C. Rodríguez-Rosario are greatly acknowledged for discussions. The (anonymous) reviewers have provided very insightful recommendations to improve the style of the original manuscript and also spotted an important technical mistake which was corrected afterwards. We acknowledge support from the Center for Excitonics, an Energy Frontier Research Center funded by the U.S. Department of Energy, Office of Science and Office of Basic Energy Sciences under Award Number DE-SC0001088. A.A.-G. acknowledges support from National Science Foundation under awards CHE-1152291 and the Corning Foundation.

\section{References}

1 Time-Dependent Density Functional Theory, in Lecture Notes in Physics, ed. M. A. L. Marques, C. A. Ullrich, F. Nogueira, A. Rubio, K. Burke and E. K. U. Gross, Springer, 2006.

2 M. A. L. Marques and E. K. U. Gross, Time-dependent density functional theory, Annu. Rev. Phys. Chem., 2004, 55, 427.

3 K. Burke, J. Werschnik and E. K. U. Gross, Time-dependent density functional theory: Past, present, and future, J. Chem. Phys., 2005, 123(6), 062206.

4 Fundamentals of Time-Dependent Density Functional Theory, ed. M. A. L. Marques, N. T. Maitra, F. M. S. Nogueira, 
E. K. U. Gross and A. Rubio, Lecture Notes in Physics, Springer, vol. 706, 2006.

5 E. Runge and E. K. U. Gross, Density-functional theory for time-dependent systems, Phys. Rev. Lett., 1984, 52(12), 997.

6 R. van Leeuwen, Mapping from densities to potentials in time-dependent density-functional theory, Phys. Rev. Lett., 1999, 82(19), 3863-3866.

7 W. Kohn and L. J. Sham, Self-consistent equations including exchange and correlation effects, Phys. Rev., 1965, 140(4A), A1133-A1138.

8 G. Vignale, Mapping from current densities to vector potentials in time-dependent current density functional theory, Phys. Rev. B: Condens. Matter Mater. Phys., 2004, 70(20), 201102.

9 M. Petersilka, U. J. Gossmann and E. K. U. Gross, Excitation energies from time-dependent density-functional theory, Phys. Rev. Lett., 1996, 76, 1212-1215.

10 M. E. Casida, Recent advances in density functional methods: Part 1, chapter Time-Dependent Density Functional Response Theory for Molecules, World Scientific, Singapore, 1995, pp. 155-192.

11 M. E. Casida, C. Jamorski, K. C. Casida and D. R. Salahub, Molecular excitation energies to high-lying bound states from time-dependent density-functional response theory: Characterization and correction of the time-dependent local density approximation ionization threshold, J. Chem. Phys., 1998, 108(11), 4439.

12 R. Baer and D. Neuhauser, Real-time linear response for time-dependent density-functional theory, J. Chem. Phys, 2004, 121(20), 9803.

13 C. A. Ullrich, Excitation energies in time-dependent (current-) density-functional theory: A simple perspective, J. Chem. Theor. Comput., 2009, 5(4), 859.

14 A. Wasserman, N. T. Maitra and K. Burke, Accurate rydberg excitations from the local density approximation, Phys. Rev. Lett., 2003, 91(26), 263001.

15 A. Castro, H. Appel, M. Oliveira, C. A. Rozzi, X. Andrade, F. Lorenzen, M. A. L. Marques, E. K. U. Gross and A. Rubio, Octopus: a tool for the application of time-dependent density functional theory, Phys. Status Solidi B, 2006, 243(11), 2465

16 S. K. Saikin, R. Olivares-Amaya, D. Rappoport, M. Stopa and A. Aspuru-Guzik, On the chemical bonding effects in the raman response: Benzenethiol adsorbed on silver clusters, Phys. Chem. Chem. Phys., 2009, 11, 9401.

17 L. Jensen and N. Govind, Excited states of dna base pairs using long-range corrected time-dependent density functional theory, J. Phys. Chem. A, 2009, 113(36), 9761.

18 T. Stein, L. Kronik and R. Baer, Reliable prediction of charge transfer excitations in molecular complexes using timedependent density functional theory, J. Am. Chem. Soc., 2009, 131(8), 2818-2820.

19 S. Tretiak, Triplet state absorption in carbon nanotubes, a td-dft study, Nano Lett., 2007, 7(8), 2201.

20 R. Improta, C. Ferrante, R. Bozio and V. Barone, The polarizability in solution of tetra-phenyl-porphyrin derivatives in their excited electronic states: a PCM/ TD-DFT study, Phys. Chem. Chem. Phys., 2009, 11, 4664.

21 A. Karolewski, T. Stein, R. Baer and S. Kümmel, Communication: Tailoring the optical gap in light-harvesting molecules, J. Chem. Phys., 2011, 134(15), 151101.

22 Y. Takimoto, F. D. Vila and J. J. Rehr, Real-time time-dependent density functional theory approach for frequency-dependent nonlinear optical response in photonic molecules, J. Chem. Phys., 2007, $127(15), 154114$.

23 X. Andrade, S. Botti, M. A. L. Marques and A. Rubio, Timedependent density functional theory scheme for efficient calculations of dynamic (hyper)polarizabilities, J. Chem. Phys., 2007, 126(18), 184106.

24 D. Rappoport, S. Shim and A. Aspuru-Guzik, Simplified sum-over-states approach for predicting resonance raman spectra application to nucleic acid bases, J. Phys. Chem. Lett., 2011, 2(11), 1254-1260.

25 E. Sagvolden, F. Furche and A. Kohn, Forster energy transfer and davydov splittings in time-dependent density functional theory: Lessons from 2-pyridone dimer, J. Chem. Theor. Comput., 2009, 5(4), 873.

26 D. Hofmann, T. Körzdörfer and S. Kümmel, Energy transfer and förster's dipole coupling approximation investigated in a real-time Kohn-Sham scheme, Phys. Rev. A, 2010, 82(1), 012509.

27 I. D'Amico and C. A. Ullrich, Dissipation through spin coulomb drag in electronic spin transport and optical excitations, Phys. Rev. B: Condens. Matter Mater. Phys., 2006, 74(12), 121303.

28 V. Turkowski, A. Leonardo and C. A. Ullrich, Timedependent density-functional approach for exciton binding energies, Phys. Rev. B: Condens. Matter Mater. Phys., 2009, 79(23), 233201.

29 C. A. Ullrich and G. Vignale, Theory of the linewidth of intersubband plasmons in quantum wells, Phys. Rev. Lett., 2001, 87(3), 037402.

30 D. L. Whitenack and A. Wasserman, Resonance lifetimes from complex densities, J. Phys. Chem. Lett., 2010, 1(1), 407-411.

31 Y. Zhou and M. Ernzerhof, Calculating the lifetimes of metastable states with complex density functional theory, J. Phys. Chem. Lett., 2012, 3(14), 1916-1920.

32 F. Furche and R. Ahlrichs, Adiabatic time-dependent density functional methods for excited state properties, J. Chem. Phys., 2002, 117(16), 7433.

33 M. Thiele, E. K. U. Gross and S. Kümmel, Adiabatic approximation in nonperturbative time-dependent densityfunctional theory, Phys. Rev. Lett., 2008, 100(15), 153004.

34 R. Baer, Prevalence of the adiabatic exchange-correlation potential approximation in time-dependent density functional theory, THEOCHEM, 2009, 914(1-3), 19. Timedependent density-functional theory for molecules and molecular solids.

35 N. T. Maitra, K. Burke and C. Woodward, Memory in timedependent density functional theory, Phys. Rev. Lett., 2002, 89(2), 023002. 
36 A. K. Rajam, I. Raczkowska and N. T. Maitra, Semiclassical electron correlation in density-matrix time propagation, Phys. Rev. Lett., 2010, 105(11), 113002.

37 M. Ruggenthaler and D. Bauer, Rabi oscillations and fewlevel approximations in time-dependent density functional theory, Phys. Rev. Lett., 2009, $102(23), 233001$.

38 J. I. Fuks, N. Helbig, I. V. Tokatly and A. Rubio, Nonlinear phenomena in time-dependent density-functional theory: What rabi oscillations can teach us, Phys. Rev. B: Condens. Matter Mater. Phys., 2011, 84, 075107.

39 I. Tavernelli, U. F. Röhrig and U. Rothlisberger, Molecular dynamics in electronically excited states using time-dependent density functional theory, Mol. Phys., 2005, 103(6-8), 963-981.

40 C. M. Isborn, X. Li and J. C. Tully, Time-dependent density functional theory ehrenfest dynamics: Collisions between atomic oxygen and graphite clusters, J. Chem. Phys., 2007, 126(13), 134307.

41 C. F. Craig, W. R. Duncan and O. V. Prezhdo, Trajectory surface hopping in the time-dependent Kohn-Sham approach for electron-nuclear dynamics, Phys. Rev. Lett., 2005, 95(16), 163001.

42 B. F. Habenicht and O. V. Prezhdo, Nonradiative quenching of fluorescence in a semiconducting carbon nanotube: A time-domain $a b$ initio study, Phys. Rev. Lett., 2008, 100(19), 197402.

43 S. A. Fischer, B. F. Habenicht, A. B. Madrid, W. R. Duncan and O. V. Prezhdo, Regarding the validity of the timedependent Kohn-Sham approach for electron-nuclear dynamics via trajectory surface hopping, J. Chem. Phys., 2011, 134(2), 024102.

44 T. Kreibich, R. van Leeuwen and E. K. U. Gross, Multicomponent density-functional theory for electrons and nuclei, Phys. Rev. A, 2008, 78(2), 022501.

45 E. Tapavicza, I. Tavernelli, U. Rothlisberger, C. Filippi and M. E. Casida, Mixed time-dependent density-functional theory/classical trajectory surface hopping study of oxirane photochemistry, J. Chem. Phys., 2008, 129(12), 124108.

46 K. Burke, R. Car and R. Gebauer, Density functional theory of the electrical conductivity of molecular devices, Phys. Rev. Lett., 2005, 94(14), 146803.

47 M. Koentopp, C. Chang, K. Burke and R. Car, Density functional calculations of nanoscale conductance, J. Phys.: Condens. Matter, 2008, 20(8), 083203.

48 M. Di Ventra and R. D'Agosta, Stochastic time-dependent current-density-functional theory, Phys. Rev. Lett., 2007, 98(22), 226403.

49 R. D'Agosta and M. Di Ventra, Stochastic time-dependent current-density-functional theory: A functional theory of open quantum systems, Phys. Rev. B: Condens. Matter Mater. Phys., 2008, 78(16), 165105.

50 D. Neuhauser and K. Lopata, Quantum drude friction for time-dependent density functional theory, J. Chem. Phys., 2008, 129(13), 134106.

51 J. Yuen-Zhou, C. Rodríguez-Rosario and A. Aspuru-Guzik, Time-dependent current-density functional theory for generalized open quantum systems, Phys. Chem. Chem. Phys., 2009, 11, 4509.

52 V. Krishna, Time-dependent density-functional theory for nonadiabatic electronic dynamics, Phys. Rev. Lett., 2009, 102(5), 053002.

53 J. Yuen-Zhou, D. G. Tempel, C. A. Rodrguez-Rosario and A. Aspuru-Guzik, Time-dependent density functional theory for open quantum systems with unitary propagation, Phys. Rev. Lett., 2010, 104(4), 043001.

54 D. G. Tempel, M. A. Watson, R. Olivares-Amaya and A. Aspuru-Guzik, Time-dependent density functional theory of open quantum systems in the linear-response regime, J. Chem. Phys., 2011, 134(7), 074116.

55 D. G. Tempel and A. Aspuru-Guzik, Relaxation and dephasing in open quantum systems time-dependent density functional theory: Properties of exact functionals from an exactly-solvable model system, Chem. Phys., 2011, (1), 130.

56 X. Zheng, F. Wang, C. Y. Yam, Y. Mo and G. H. Chen, Timedependent density-functional theory for open systems, Phys. Rev. B: Condens. Matter Mater. Phys., 2007, 75(19), 195127.

57 X. Zheng, G. H. Chen, Y. Mo, S. K. Koo, H. Tian, C. Y. Yam and Y. J. Yan, Time-dependent density functional theory for quantum transport, J. Chem. Phys., 2010, 133(11), 114101.

58 S. Wen, S. Koo, C. Y. Yam, X. Zheng, Y. J. Yan, Z. Su, K. Fan, L. Cao, W. Wang and G. H. Chen, Time-dependent current distributions of a two-terminal carbon nanotube-based electronic device, J. Phys. Chem. Lett., 2011, 115(18), 5519.

$59 \mathrm{X} . \mathrm{Li}$ and J. C. Tully, Ab initio time-resolved density functional theory for lifetimes of excited adsorbate states at metal surfaces, Chem. Phys. Lett., 2007, 439(1-3), 199.

$60 \mathrm{~J}$. Dalibard, Y. Castin and K. Mølmer, Wave-function approach to dissipative processes in quantum optics, Phys. Rev. Lett., 1992, 68(5), 580-583.

61 A. A. Kocherzhenko, F. C. Grozema and L. D. A. Siebbeles, Single molecule charge transport: from a quantum mechanical to a classical description, Phys. Chem. Chem. Phys., 2011, 13, 2096.

62 D. J. Masiello and G. C. Schatz, On the linear response and scattering of an interacting molecule-metal system, J. Chem. Phys., 2010, 132(6), 064102.

63 C. T. Chapman, W. Liang and X. Li, Open-system electronic dynamics and thermalized electronic structure, J. Chem. Phys., 2011, 134(2), 024118.

64 R. D'Agosta and M. Di Ventra, Comment on "Time-dependent current-density functional theory for generalized open quantum systems" by J. Yuen-Zhou, C. Rodriiguez-Rosario and A. Aspuru-Guzik. e-print arXiv:0911.3932.

65 R. D'Agosta and M. Di Ventra, Foundations of stochastic time-dependent current-density functional theory for open quantum systems: potential pitfalls and rigorous results, Phys. Rev. B, 2013, 87, 155129.

66 N. Bushong and M. Di Ventra, The decay of excited he from stochastic density-functional theory: a quantum measurement theory interpretation, J. Phys.: Condens. Matter, 2008, 20(39), 395214.

67 R. Gebauer and R. Car, Current in open quantum systems, Phys. Rev. Lett., 2004, 93(16), 160404. 
68 A. Bodor and L. Diósi, Conserved current in markovian open-quantum systems, Phys. Rev. A, 2006, 73(6), 064101.

69 R. Gebauer, S. Piccinin and R. Car, Quantum collision current in electronic circuits, ChemPhysChem, 2005, 6(9), 1727.

70 C. Meier and D. J. Tannor, Non-markovian evolution of the density operator in the presence of strong laser fields, J. Chem. Phys., 1999, 111(8), 3365.

71 H.-P. Breuer and F. Petruccione, The Theory of Open Quantum Systems, Oxford University Press, 2002.

72 R. Zwanzig, Nonequilibrium Statistical Mechanics, Oxford University Press, 2001.

73 V. May and O. Kuhn, Charge and Energy Transfer Dynamics in Molecular Systems, Wiley-VCH, 2004.

74 A. Nitzan, Chemical Dynamics in Condensed Phases, Oxford University Press, 2006.

75 A. Kossakowski, On quantum statistical mechanics of nonhamiltonian systems, Rep. Math. Phys., 1972, 3(4), 247-274.

76 V. Gorini, A. Kossakowski and E. C. G. Sudarshan, Completely positive dynamical semigroups of n-level systems, J. Math. Phys., 1976, 17(5), 821.

77 G. Lindblad, On the generators of quantum dynamical semigroups, Commun. Math. Phys., 1976, 48, 119.

78 B. Schumacher and M. Westmoreland, Quantum Processes, Systems, and Information, Cambridge University Press, 2010.

79 L. Diosi, Stochastic pure state representation for open quantum systems, Phys. Lett. A, 1986, 114(8-9), 451.

80 H.-P. Breuer, W. Huber and F. Petruccione, Stochastic wavefunction method versus density matrix: a numerical comparison, Comput. Phys. Commun., 1997, 104(1-3), 46.

81 M. B. Plenio and P. L. Knight, The quantum-jump approach to dissipative dynamics in quantum optics, Rev. Mod. Phys., 1998, 70(1), 101.

82 A. Suarez, R. Silbey and I. Oppenheim, Memory effects in the relaxation of quantum open systems, J. Chem. Phys., 1992, 97(7), 5101.
83 Y. C. Cheng and R. J. Silbey, Markovian approximation in the relaxation of open quantum systems, J. Phys. Chem. B, 2005, 109(45), 21399-21405.

84 P. Gaspard and M. Nagaoka, Slippage of initial conditions for the redfield master equation, J. Chem. Phys., 1999, 111(13), 5668.

85 B. Palmieri, D. Abramavicius and S. Mukamel, Lindblad equations for strongly coupled populations and coherences in photosynthetic complexes, J. Chem. Phys., 2009, 130(20), 204512.

86 P. Rebentrost, M. Mohseni, I. Kassal, S. Lloyd and A. Aspuru-Guzik, Environment-assisted quantum transport, New J. Phys., 2009, 11(3), 033003.

87 J. Wilkie, Positivity preserving non-markovian master equations, Phys. Rev. E, 2000, 62(6), 8808.

88 D. Kohen, C. C. Marston and D. J. Tannor, Phase space approach to theories of quantum dissipation, J. Chem. Phys., 1997, 107(13), 5236.

89 W. T. Pollard and R. A. Friesner, Solution of the redfield equation for the dissipative quantum dynamics of multilevel systems, J. Chem. Phys., 1994, 100(7), 5054.

90 I. Kondov, U. Kleinekathöfer and M. Schreiber, Efficiency of different numerical methods for solving redfield equations, J. Chem. Phys., 2001, 114(4), 1497.

91 D. Egorova, M. Thoss, W. Domcke and H. Wang, Modeling of ultrafast electron-transfer processes: validity of multilevel redfield theory, J. Chem. Phys., 2003, 119(5), 2761.

92 A. Perdomo, L. Vogt, A. Najmaie and A. Aspuru-Guzik, Engineering directed excitonic energy transfer, Appl. Phys. Lett., 2010, 96(9), 093114.

93 D. G. Tempel, J. Yuen-Zhou and A. Aspuru-Guzik, Open Quantum Systems: Density Matrix Formalism and Applications, in Fundamentals of Time-Dependent Density Functional Theory, ed. M. A. L. Marques, N. T. Maitra, F. M. S. Nogueira, E. K. U. Gross and A. Rubio, Lecture Notes in Physics, Springer, 2012, vol. 837, pp. 211-229. 